\title{
DNA Strand Breaks in Mitotic Germ Cells of Caenorhabditis elegans Evaluated by Comet Assay
}

\author{
Sojin Park ${ }^{1,2}$, Seoyun Choi', and Byungchan Ahn ${ }^{1, *}$
}

\begin{abstract}
DNA damage responses are important for the maintenance of genome stability and the survival of organisms. Such responses are activated in the presence of DNA damage and lead to cell cycle arrest, apoptosis, and DNA repair. In Caenorhabditis elegans, double-strand breaks induced by DNA damaging agents have been detected indirectly by antibodies against DSB recognizing proteins. In this study we used a comet assay to detect DNA strand breaks and to measure the elimination of DNA strand breaks in mitotic germline nuclei of $C$. elegans. We found that $C$. elegans brc-1 mutants were more sensitive to ionizing radiation and camptothecin than the N2 wild-type strain and repaired DNA strand breaks less efficiently than N2. This study is the first demonstration of direct measurement of DNA strand breaks in mitotic germline nuclei of $C$. elegans. This newly developed assay can be applied to detect DNA strand breaks in different $C$. elegans mutants that are sensitive to DNA damaging agents.
\end{abstract}

\section{INTRODUCTION}

The integrity of DNA is continuously challenged by a variety of exogenous and endogenous DNA-damaging agents. Eukaryotes have evolved to possess a DNA damage response (DDR) that triggers cell cycle arrest, apoptosis, and DNA repair to protect the cell and ameliorate the threat to the organism.

The DDR signaling cascade is highly conserved in Caenorhabditis elegans. The DDR in the $C$. elegans germline involves the coordinated induction of DNA repair, cell cycle arrest, and apoptotic processes (Craig et al., 2012; Lemmens and Tijsterman, 2011). ATM-1 and ATL-1 are typical kinases of the DDR pathway that phosphorylate a large number of substrates in response to DNA damage (Garcia-Muse and Boulton, 2005; Lee et al., 2010). MRE-11, RAD-50, HUS-1, and MRT-2 proteins are required for the function of these kinases (Ahmed and

${ }^{1}$ Department of Life Sciences, University of Ulsan, Ulsan 44610, Korea, ${ }^{2}$ Present address: Green Cross Genome, Yongin 16924, Korea

*Correspondence: bbccahn@mail.ulsan.ac.kr

Received 22 July, 2015; revised 23 November, 2015; accepted 23 November, 2015; published online 23 February, 2016

Keywords: Caenorhabditis elegans, comet assay, DNA damage, DNA repair, DNA strand break
Hodgkin, 2000; Chin and Villeneuve, 2001; Hofmann et al., 2002). Through the activation of downstream kinases CHK-1 and CHK-2, signaling is amplified down to the activation of effector molecules (Kalogeropoulos et al., 2004; Lee et al., 2010; MacQueen and Villeneuve, 2001).

DNA double-strand breaks (DSBs) can be induced either directly by exposure to ionizing irradiation (IR) or indirectly by the topoisomerase I inhibitor camptothecin (CPT), which causes replication fork stalling and collapse in actively cycling cells. DDR proteins for DSBs in mammalian cells are the kinases ATM/ATR, the sensing complex MRE11-RAD50-NBS1, and CHK2 (Langerak and Russell, 2011). The presence of DSBs is indirectly detected by immunostaining the proteins $\gamma-\mathrm{H} 2 \mathrm{AX}$ or RAD51 (Paull et al., 2000; Tarsounas et al., 2004). H2AX is phosphorylated (gamma-H2AX) following exposure to IR and is densely localized around DSBs. Since RAD51 plays an essential role in homologous recombination for DSB repair in mammalian cells, RAD51 foci formation is thought to represent the presence of DSBs. Thus, immunostaining of $\gamma-\mathrm{H} 2 \mathrm{AX}$ and RAD51 is useful for visualizing the localization of DSBs. RAD51 foci formation has also been used to detect the sites of DSBs following IR in C. elegans (Wicky et al., 2004).

Another method used to detect DSBs and their repair is the comet assay, a rapid and quantitative technique by which damaged or broken pieces of DNA are measured at the level of individual cells (Olive and Banath, 2006). Increased comet tails indicate the induction of DNA strand breaks. Initially, DNA strand breaks induced by IR or UV irradiation were detected in human blood cells by the comet assay (Lankinen et al., 1996; Olive et al., 1990), and DNA strand breaks induced by other agents such as CPT were subsequently detected (Godard et al., 2002). To date, there is only one report of use of the comet assay in $C$. elegans to investigate the effect of nicotine on cultured embryonic cells (Sobkowiak and Lesicki, 2009), but the authors did not investigate mitotic germline nuclei to observe DNA strand breaks induced by IR or CPT.

The identified C. elegans protein BRC-1 (C36A4.8), a protein of 596 amino acids, is an ortholog of human BRCA1. Studies of the effects of depletion of $b r c-1$ have described IR sensitivity and enhanced levels of germ cell death and DNA fragmentation after IR, suggesting that BRC-1 is involved in DSB repair by homologous recombination (HR) (Boulton et al., 2004). A role of BRC-1 in the repair pathway of inter-sister meiotic DSBs is also suggested (Adamo et al., 2008). However, the defect in DSB repair has not been well investigated at the DNA level. In this 
study, we used the comet assay to investigate DNA strand breakage and repair in the N2 wild-type strain and brc-1 mutant which was chose as a suitable DNA repair mutant. .

\section{MATERIAL AND METHODS}

\section{Strains}

The C. elegans Bristol N2 wild-type strain was maintained on nematode growth media (NGM) plates at $20^{\circ} \mathrm{C}$ as described previously (Lee et al., 2010). The C. elegans mutant strain brc1(tm1145) was obtained from the Caenorhabditis Genetics Center (USA).

\section{Treatment with lonizing radiation and camptothecin} L4-stage animals on NGM plates were irradiated using a ${ }^{137} \mathrm{Cs}$ source (gamma cell 3000 ELAN) with a rate of $321 \mathrm{rad} / \mathrm{min}$ on ice. L4-stage animals were grown on NGM plates containing 0 , $1,2,5,10,20$, or $40 \mu \mathrm{M} \mathrm{CPT}$ (Sigma-Aldrich) for $24 \mathrm{~h}$ at $20^{\circ} \mathrm{C}$.

\section{Measurement of embryonic survival}

IR-treated worms were allowed to lay eggs. After $24 \mathrm{~h}$, the number of unhatched eggs was counted. L4-stage animals were grown on NGM plates containing $0,1,2,5,10,20$, or 40 $\mu \mathrm{M} \mathrm{CPT}$ for $24 \mathrm{~h}$ and then transferred to CPT-free plates with $E$. coli OP50, where eggs were laid. After $24 \mathrm{~h}$, the numbers of hatched and unhatched eggs were counted. The embryonic survival percentage was calculated by dividing the number of hatched eggs by the total number of eggs laid.

\section{Comet assays}

For the glyoxal-treated comet assay, mitotic germline nuclei were treated with glyoxal to denature the DNA (Hyun et al., 2008). The dissected gonads (30-40 worms) were cut to expose the mitotic tip regions. The mitotic compartments were mixed with glass beads (212-300 $\mu \mathrm{m}$ in diameter) in a microcentrifuge tube and disrupted in a mini bead-beater (company) at $500 \times g$ for $10 \mathrm{~s}$. The supernatants containing mitotic nuclei were separated by centrifugation at $100 \times g$ for $10 \mathrm{~s}$ and mixed with low-melting point agarose. The mixture was loaded on an agar-coated microscope slide. The slide was immersed in icecold lysis solution (2.5 M NaCl, $100 \mathrm{mM}$ EDTA, $10 \mathrm{mM}$ Tris-Cl: $\mathrm{pH} 8.4,1 \%$ Triton $\mathrm{X}-100,10 \% \mathrm{DMSO})$ at $4^{\circ} \mathrm{C}$ for $15 \mathrm{~min}$ and then incubated with $1 \mathrm{M}$ glyoxal in lysis buffer $\left(1: 1\right.$ ratio) at $4^{\circ} \mathrm{C}$ for $1 \mathrm{~h}$. Electrophoresis was performed at 50 volts for $90 \mathrm{~min}$ in TBE buffer. After electrophoresis, the slides were washed with deionized $\mathrm{H}_{2} \mathrm{O}$ and then stained with DAPI (4,6-diamidino-2phenylindole, $1 \mu \mathrm{g} / \mathrm{ml}$, Sigma-Aldrich) or SYBR Gold (1:10,000 dilution, Molecular Probes) for $10 \mathrm{~min}$. Observations were performed under an epifluorescence microscope (Carl Zeiss Axioskop2 plus). Quantitation was performed on 60-100 nuclei from each gel using Comet Assay IV software (Perceptive Instruments, Ltd.).

Neutral comet assays were performed by a slight modification of the glyoxal-treated comet assay: after the lysis step, instead of glyoxal treatment, the slides were incubated with RNase A (1 mg/ml, Sigma-Aldrich) and proteinase $\mathrm{K}(32 \mathrm{U} / \mathrm{ml}$, Roche) for $1 \mathrm{~h}$ at $37^{\circ} \mathrm{C}$ and then electrophoresis was performed at 20 volts for 90 min in TBE buffer. After electrophoresis, the slides were stained and analyzed as above. We used Olive tail moment in this study because it is considered independent of comet shape and is the best-established parameter for this assay, with less variability than tail length and tail DNA content.

\section{Immunostaining}

The gonads of IR-irradiated worm were dissected $1 \mathrm{~h}$ after irra-

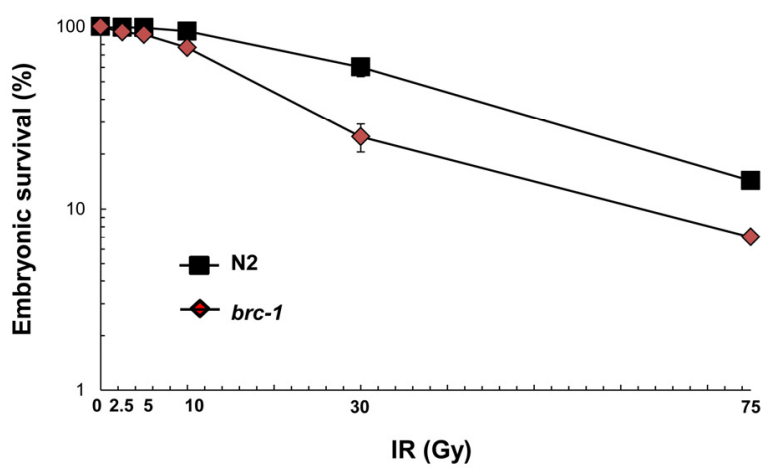

Fig. 1. The Percent survival of embryo survival after exposure to $I R$. $\mathrm{N} 2$ and brc-1 $(\mathrm{tm} 1145)$ mutants were irradiated at the indicated doses $(0,2.5,5,10,30,75 \mathrm{~Gy})$ on ice and then transferred to NGM plates with $E$. coli OP50, where eggs were laid. Hatching percentages were measured. Error bars indicate SEM.

diation. The gonads of CPT-treated worms were dissected $24 \mathrm{~h}$ after treatment. The gonads were extruded, fixed in $4 \%$ paraformaldehyde, and immunostained as described previously (Garcia-Muse and Boulton, 2005; Hyun et al., 2012). Briefly, the gonads were blocked with PBSBT (1X PBS, $0.5 \%$ bovine serum albumin, $0.1 \%$ Triton $X-100$ ) containing $2 \%$ non-fat milk at $4^{\circ} \mathrm{C}$ overnight. The gonads were incubated with primary antibody (1:1,000 dilution for anti-RAD-51, a polyclonal antibody generated with $\mathrm{N}$-terminal 103 amino acids in rabbit) in humid chambers for $16 \mathrm{~h}$ at $4^{\circ} \mathrm{C}$ and then with Alexa Fluor 488conjugated goat anti-rabbit secondary antibody (Molecular Probes) for $1 \mathrm{~h}$ at room temperature in dark conditions. After staining with DAPI, the gonads were mounted on a $1 \%$ agarose pad and observed under an epifluorescence microscope (Carl Zeiss Axioskop2 plus).

\section{RESULTS}

\section{Detection of DNA strand breaks induced by IR in C. elegans}

\section{Embryonic survival following ionizing radiation}

Since $C$. elegans brc-1(RNAi) animals have been shown to be radiation sensitive (Boulton et al., 2004), we examined the sensitivity of brc-1(tm1145) mutants to IR. The mutants were treated with ${ }^{137} \mathrm{Cs} \gamma$-rays at a dose of 0-75 Gy. The hatching percent of laid eggs from IR-treated worms decreased with increasing doses of IR, and the reduction in hatching percentage of brc-1 mutants was greater than that of wild-type N2, indicating that the brc-1 mutants are radiation sensitive (Fig. 1). At $30 \mathrm{~Gy}, \mathrm{~N} 2$ showed $60 \%$ survival whereas the brc-1 mutant showed $25 \%$ survival (Fig. 1). This dose was chosen for subsequent experiments. Since the primary determinant of IR sensitivity is the efficiency of DNA double-strand break (DSB) repair, DNA strand breaks induced by IR and the DNA repair of DNA strand breaks were examined in brc-1 mutants and wild-type N2.

DSBs accumulate in the brc-1(tm1145) mutant after IR DNA repair defects have been observed in brc-1(RNAi) animals (Boulton et al., 2004) and the defect in brc-1(tm1145) mutants has been evaluated by the formation of nuclear RAD-51 foci after IR (Polanowska et al., 2006) since RAD-51 plays an essential role in homologous recombination (Alpi et al., 2003; Petalcorin et al., 2007). However, IR-induced DSBs and repair 
A

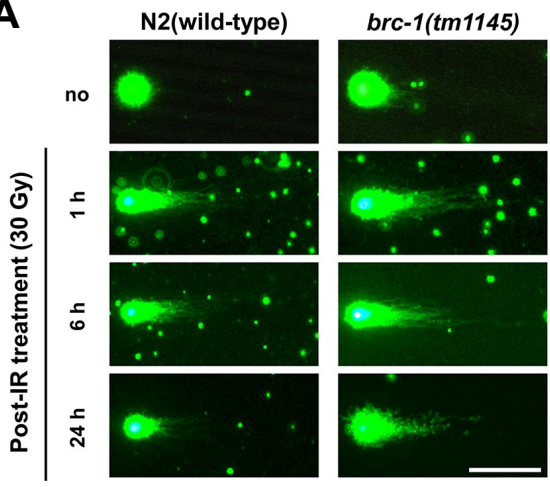

C

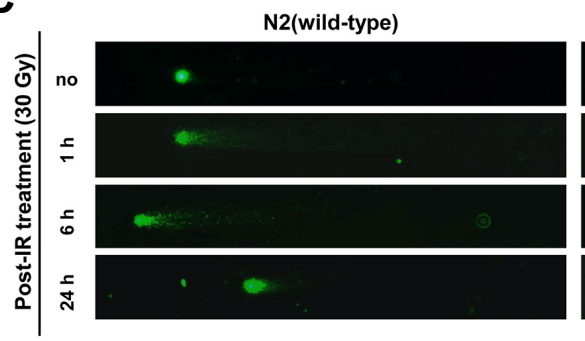

D

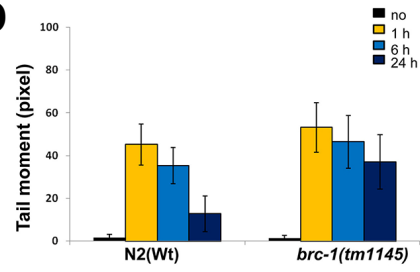

B
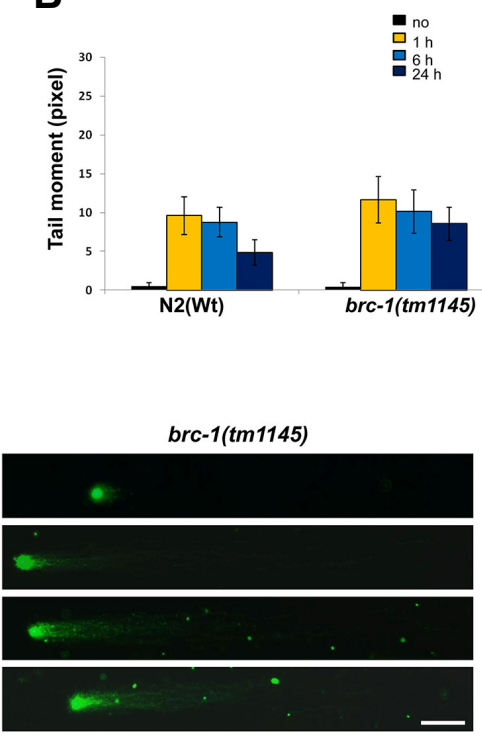

no
$1 \mathrm{~h}$
$6 \mathrm{~h}$

Fig. 2. Analysis of Comet assay. Worms were irradiated with 30 Gy of IR and postincubated for various recovery times. Comet slides were prepared, lysed, treated with glyoxal, and electrophoresed in a neutral buffer. (A) Comet images were captured using an epifluorescence microscope (Carl Zeiss), Scale bar, $50 \mu \mathrm{m}$. (B) The tail moment of comets in (A) was analyzed using Comet Assay IV software. Comet slides were prepared, lysed, treated with RNase and proteinase $\mathrm{K}$, and electrophoresed in a neutral buffer. (C) Comet images were captured using an epifluorescence microscope (Carl Zeiss), Scale bar, $50 \mu \mathrm{m}$. (D) The tail moment of comets in (C) was analyzed using Comet Assay IV software.

kinetics in brc-1 mutants have not been directly examined. We examined DNA strand breakage and repair using the comet assay, in which the extent of DNA strand breakage is assessed by DNA migration in the comet tail following irradiation with 30 Gy. Representative images of glyoxal comets are shown in Fig. $2 \mathrm{~A}$. Comet tails were observed at $1 \mathrm{~h}$ after IR, indicating that DNA strand breaks were induced by IR. The analysis of tail moments in 100 comets at recovery time of $24 \mathrm{~h}$ after IR revealed that $55 \%$ of the DNA strand breaks were repaired in N2, whereas only $27 \%$ of the DNA strand breaks were repaired in brc-1 mutants (Fig. 2B ).

The neutral comet assay was also performed to specifically examine DSB and repair. Comet tails were observed at $1 \mathrm{~h}$ after IR (Fig. 2C), indicating that DSBs were induced by IR. The analysis of tail moments in 100 comets at recovery time of $24 \mathrm{~h}$ after IR revealed that $73 \%$ of the DSBs were repaired in N2, compared with $30 \%$ in brc-1 mutants (Fig. 2D).

The tail moments in two assays were different. The extent of repair of N2 measured by the glyoxal-comet assay (Figs. 2B and 2D) was lower than that by the neutral comet assay, indicating that unrepaired single-strand breaks reflect the difference. The extent of repair brc-1 mutants at recovery time of $24 \mathrm{~h}$ is similar, indicating that unrepaired DSBs may reflect the extent of repair. Taken together, these data support a previous finding that brc-1 mutants are defective in DSB repair (Boulton et al., 2004).
Detection of DNA strand breaks induced by camptothecin in C. elegans

Embryonic survival following camptothecin treatment CPT, a selective inhibitor of topoisomerase I (TOP1), stabilizes TOP1-DNA covalent complexes. Collisions between the replication fork migrating along the DNA and a trapped TOP1-DNA covalent complex result in irreversible replication fork arrest and DSB formation at the fork (Pommier, 2006; Ryan et al., 1991). Since the sensitivity of brc-1 mutants to CPT has not been reported, we first examined the embryonic survival of brc-1 mutants after treatment with the indicated concentrations of CPT for $24 \mathrm{~h}$ (Fig. 3). The hatching percentage of laid eggs from the CPT-treated brc- 1 mutants was greatly reduced after CPT treatment. At $5 \mu \mathrm{M}$ CPT, the N2 strain showed $60 \%$ survival, compared with $22 \%$ for the brc-1 mutant. We selected a concentration of $5 \mu \mathrm{M}$ CPT for the next experiments.

DSBs accumulate in the brc-1(tm1145) mutant after CPT treatment

We have previously shown that CPT induces DSBs in wild-type N2 by demonstrating an increase in the numbers of germline nucleus showing RAD-51-positive foci (manuscript in preparation). RAD-51 foci were also detected in mitotic nuclei of N2 and brc-1 after CPT treatment (Fig. S2). We examined whether CPT-induced RAD-51 foci formation reflects DNA strand breaks 


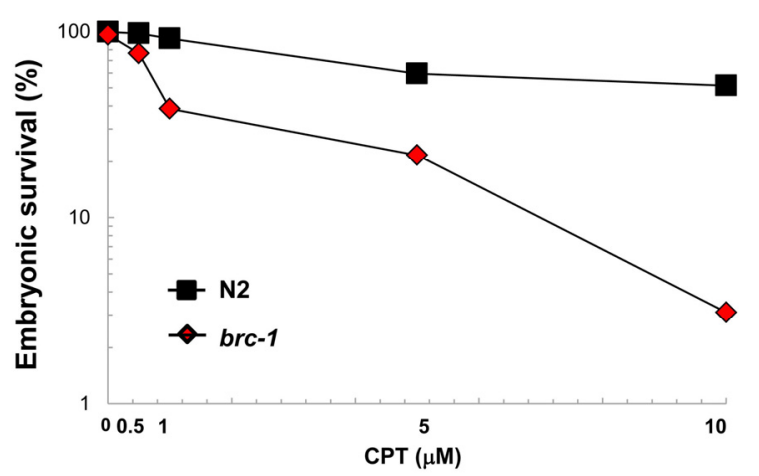

Fig. 3. The Percent survival of embryo survival after treatment with CPT. N2 and brc-1(tm1145) mutants were treated with the indicated concentrations of CPT for $24 \mathrm{~h}$ and then transferred to CPT-free plates with E. coli OP50, where eggs were laid. Hatching percentages were measured. Error bars indicate SEM.

in germline nuclei in $C$. elegans and investigated the repair of CPT-induced DNA strand breaks using the comet assay.

The glyoxal comet assay was first performed to confirm the presence of DNA strand breaks. Representative images are shown (Fig. 4A). There was an increase in CPT-induced DNA strand breaks compared with non-damaged controls in both wild-type N2 and brc-1 mutants (compare the data of no (no CPT-treated worms) with the data of $0 \mathrm{~h}$ in Figs. $4 \mathrm{~A}$ and $4 \mathrm{~B}$ ). The analysis of tail moments for DNA strand breaks observed at different recovery times after CPT treatment revealed that $60 \%$ of the DNA strand breaks were removed at $24 \mathrm{~h}$ after CPT treatment in $\mathrm{N} 2$, whereas the extent of DNA strand breakage remained unchanged in brc-1 mutants (Fig. 4B). The N2 worms showed little removal of DNA strand breaks before $12 \mathrm{~h}$ and seemed to start remove after that time. These results suggest that repair of CPT-induced DNA strand breaks is defective in brc-1 mutants.

The neutral comet assay was also performed to assess DSBs induced by CPT. Representative images are shown (Fig. 4C). There was an increase in CPT-induced DSBs compared with non-damaged controls in both wild-type N2 and brc-1 mutants (compare the data of no (no CPT-treated worm) with the data of $0 \mathrm{~h}$ in Figs. 4C and 4D). The analysis of tail moments in 100 comets of N2 at each time points after CPT treatment revealed that the tail lengths of DSBs were gradually decreased and greatly diminished (85\%) at $24 \mathrm{~h}$, but remained long (Fig. 4D) in brc-1 mutants.

The tail moments in two assays were different. The extent of repair of N2 measured by the glyoxal-comet assay (Figs. 4B and 4D) was lower than that by the neutral comet assay, indicating that unrepaired single-strand breaks reflect the difference. Interestingly, DSBs were further generated during recovery times in brc-1 mutants when treated with CTP. These data further indicate that brc-1 mutants are defective in DSB repair and implicate a role for BRC-1 in the DSB repair process.

\section{DISCUSSION}

In this study, we have investigated DNA DSB induction and repair in wild-type N2 and bcr-1 mutants using the comet assay. Although both RAD-51 foci formation and chromosome fragmentation have been applied for the detection of DSB induction and DSB repair in mitotic germline nuclei $C$. elegans, use of the comet assay has not previously been reported in those nuclei. In this study we successfully detected the extent of DNA strand breaks in $C$. elegans using the comet assay.

We chose a brc-1 mutant as a suitable DNA repair mutant for comparison of the detection of DNA strand breakage and repair with that of wild-type. BRC-1 was previously identified as the $C$. elegans ortholog of BRCA1, which is required for DSB repair in mammalian cells, and $b r c-1$ depleted animals were shown to be sensitive to DSB-inducing IR, suggesting a role of BRC-1 in $\mathrm{HR}$ in C. elegans (Boulton et al., 2004). BRC-1 forms a complex with BRD-1 and is recruited to sites of DNA damage (Boulton et al., 2004; Polanowska et al., 2006), consistent with the role of BRCA1 in mammalian cells (Meza et al., 1999; Moynahan et al., 1999; Scully et al., 1999). BRC-1 is also required for HR between sister chromatids in meiotic cells (Adamo et al., 2008). In this study, we showed that brc-1 mutants were sensitive to IR and CPT treatments, suggesting that $\mathrm{BRC}-1$ may be involved in processing DNA damage induced by these treatments.

The comet assay, also known as single-cell gel electrophoresis, can detect DNA damage and repair kinetics at the level of a single cell and has been shown to be a suitable tool for studying the induction and repair of radiation-induced DSBs (Olive and Banath, 2006). The alkaline comet, in which DNA is mobilized under alkaline conditions for DNA denaturation, detects both single-stranded DNA breaks and DSBs, without distinguishing between the two. The neutral comet, in which DNA migrates under neutral conditions, detects only DSBs.

There is only one previous report of use of the comet assay in C. elegans. Sobkowiak et al. used the alkaline comet assay to investigate the in vitro response of cultured embryonic cells of C. elegans to nicotine (Sobkowiak and Lesicki, 2009). Their study suggested that the comet assay could be performed with other cells from $C$. elegans; however, the authors did not investigate the comet assay using mitotic germline nuclei. In this study, we physically isolated mitotic germline nuclei of $C$. elegans as described in the Methods and successfully carried out comet assays.

We treated germline nuclei with glyoxal instead of alkaline treatment because the presence of alkali-labile sites in DNA can result in the introduction of additional single-strand breaks upon alkali treatment during DNA sample processing. Glyoxal is known to covalently bind to only solution-exposed guanine bases (Broude and Budowsky, 1973). We have previously used glyoxal in DNA hybridization of $C$. elegans genomic DNA (Hyun et al., 2008) because the $C$. elegans genomic DNA was detected to be degraded under alkaline electrophoresis condition (unpublished data). There was a clear difference in tail length between untreated germline nuclei from either N2 or brc-1 mutants and IR-treated germline nuclei, indicating that IR induces DNA strand breaks. CPT-induced DNA strand breaks were also detected in N2 and brc-1 mutants. Unchanged tail length of CPT-induced DNA strand breaks in brc-1 mutants may present that initial DNA strand breaks generated by CPT treatment were not removed and no more DNA strand break was produced during recovery time, indicating defects in repair of DNA strand breaks. Consistent with these findings, DNA strand breaks induced by IR or CPT can be detected by the alkaline comet assay or glyoxal comet assay in mammalian cells (Godard et al., 2002; Lankinen et al., 1996; Pachkowski and Nakamura, 2013).

To attain high enough sensitivity under the neutral comet assay, germline nuclei were treated with ribonuclease A and proteinase $\mathrm{K}$ to obtain relatively pure DNA free from RNA and most proteins (Singh and Khan, 1995). In the wild-type N2 strain, 
A

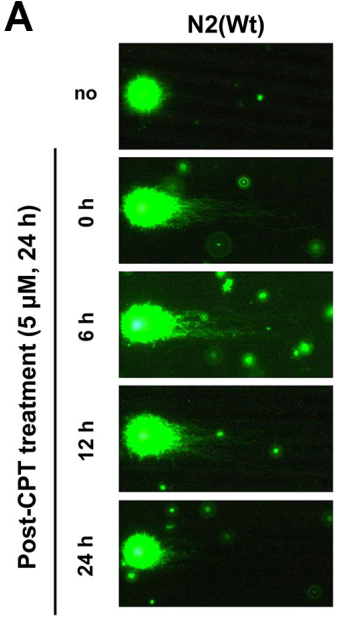

B

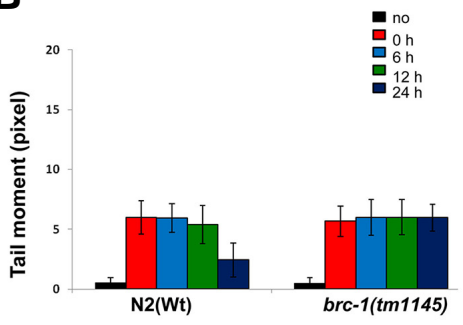

C

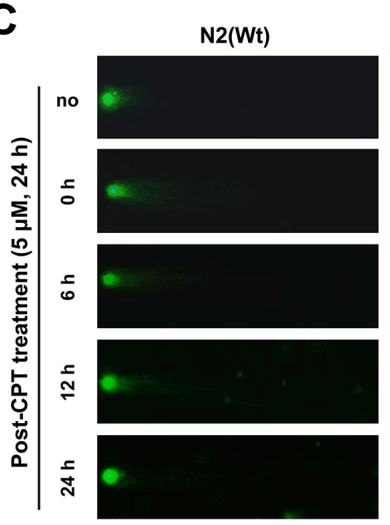

Fig. 4. Analysis of Comet assay. Worms were treated with $5 \mu \mathrm{M} \mathrm{CPT}$ for $24 \mathrm{~h}$ and postincubated on new fresh NGM plates for various recovery times. Comet slides were prepared, lysed, treated with glyoxal, and electrophoresed in a neutral buffer. (A) Comet images were captured using an epifluorescence microscope (Carl Zeiss), Scale bar, $50 \mu \mathrm{m}$. (B) The tail moment of comets in (A) was analyzed using Comet Assay IV software. Comet slides were prepared, lysed, treated with RNase and proteinase $\mathrm{K}$, and electrophoresed in a neutral buffer. (C) Comet images were captured using an epifluorescence microscope (Carl Zeiss), Scale bar, $50 \mu \mathrm{m}$. (D) The tail moment of comets in (C) was analyzed using Comet Assay IV software. no, no CPT-treated worms.
DSBs were detected following treatment with IR or CPT, which is in agreement with studies using the neutral comet assay in human cells (Lankinen et al., 1996; Olive et al., 1990). Comet tails were barely detected under the neutral comet assay without treatments with ribonuclease $\mathrm{A}$ and proteinase $\mathrm{K}$ (unpublished data). The use of ribonuclease $A$ and proteinase $K$ in this study improved sensitivity of the neutral comet assay. Since DNA strand breaks detected by the glyoxal comet assay come from both single-strand breaks and double-strand breaks, greater tail moments would be expected than those from only double-strand breaks. However, the tail moment detected by the neutral comet assay was greater than that by the glyoxal comet assay. Because sample treatment and the electrophoresis conditions such as volts, currents, and times are different in two assays, it is hard to compare the tail moments directly. Nonetheless, these experiments provided evaluation of the comet assays as a reliable tool to study DNA strand breaks in $C$. elegans.

DNA damage levels, as measured by tail moment, remained high in brc-1 nuclei at $24 \mathrm{~h}$ after IR, compared to $1 \mathrm{~h}$. DSBs were increased after CPT treatment during recovery times in brc-1 mutants. CPT-induced DNA strand breaks can be converted to DSBs by DNA replication in S-phase and the DSBs can be leveled off at a certain time if not repaired. These results suggest that DSB repair is defective in brc-1. Similarly, human cells deficient in BRCA1 are more sensitive to ionizing radiation. DSB repair kinetics measured by the comet assay or pulsed field gel electrophoresis in BRCA1 mutants or BRCA1 missense mutants showed that these mutants failed to repair DSBs efficiently (Chen et al., 2014; Scully et al., 1999).

When mammalian cells are exposed to IR, aggregates of repair proteins called nuclear foci appear within their nuclei (Bekker-Jensen et al., 2006). These foci contain proteins involved in HR including RAD51, RAD52, and RAD54, and form in response to DNA damage. Thus, it is thought that they represent sites at which recombinational repair reactions occur (Essers et al., 2002). RAD51 foci formation has served as an indicator of the induction of radiation-induced DSBs and DNA repair (Miyazaki et al., 2004; van Veelen et al., 2005). In the study of $C$. elegans DSB repair, DSBs induced by IR have been detected by RAD-51 foci in mitotic germline nuclei of $C$. elegans and DSBs formed during meiotic recombination have been also detected by RAD-51 foci (Adamo et al., 2008; Boulton et al., 2004; Garcia-Muse and Boulton, 2005).

We analyzed RAD-51 foci formation over time after exposure to IR. In wild-type N2, the percentage of germline nuclei showing RAD-51-positive foci decreased with increasing postirradiation time (Fig. S1). In contrast, the decrease in foci in brc1 was less than that in N2, indicating less efficient DSB repair. Even though BRC-1 deficiency is associated with a DSB repair defect, brc-1 mutants remain competent for RAD-51 foci formation after IR (Boulton et al., 2004), indicating that that BRC-1 is dispensable for the recruitment of RAD- 51 to sites of DSBs (Polanowska et al., 2006). Our results suggest that BRC-1 may 
be involved in a step after RAD-51 binds to DSBs. In mammalian cells, BRCA1 aggregates into nuclear foci after exposure to IR and appears to associate with RAD51 (Powell and Kachnic, 2003). However, radiation-induced RAD51 foci still form in BRCA1-deficient tumor cells (Nakada et al., 2012; Scully et al., 1999).

IR-induced DSBs in mammalian cells have been also detected by immuno staining of $\gamma-\mathrm{H} 2 \mathrm{AX}$, which is a marker for DNA strand breaks (Paull et al., 2000). DSB repair kinetics measured by the comet assay correlated with $\gamma-\mathrm{H} 2 \mathrm{AX}$ foci association. Quantitative studies further indicated that the extent of $\gamma-\mathrm{H} 2 \mathrm{AX}$ formation is proportional to the number of DSBs and that their appearance and disappearance correlate very well with the kinetics of DSB repair (Balajee and Geard, 2004; Olive, 2011). Since antibodies against $\gamma-\mathrm{H} 2 \mathrm{AX}$ in $C$. elegans are not available, we could not examine the formation of $\gamma-\mathrm{H} 2 \mathrm{AX}$ foci in N2 or brc-1 mutants.

In summary, we have shown that the dissected mitotic germline region can be used for the comet assay in $C$. elegans and that DNA strand breaks can be detected by glyoxal or neutral comet assays. These assays can be used for the detection of DNA strand breaks induced by other DNA damaging agents and for measuring DNA repair kinetics in $C$. elegans.

Note: Supplementary information is available on the Molecules and Cells website (www.molcells.org).

\section{ACKNOWLEDGMENTS}

This work was supported by the grant of University of Ulsan (2015) to B. Ahn.

\section{REFERENCES}

Adamo, A., Montemauri, P., Silva, N., Ward, J.D., Boulton, S.J., and La, V.A. (2008). BRC-1 acts in the inter-sister pathway of meiotic double-strand break repair. EMBO Rep. 9, 287-292.

Ahmed, S., and Hodgkin, J. (2000). MRT-2 checkpoint protein is required for germline immortality and telomere replication in $C$. elegans. Nature 403, 159-164.

Alpi, A., Pasierbek, P., Gartner, A., and Loidl, J. (2003). Genetic and cytological characterization of the recombination protein RAD-51 in Caenorhabditis elegans. Chromosoma 112, 6-16.

Balajee, A.S., and Geard, C.R. (2004). Replication protein A and gamma-H2AX foci assembly is triggered by cellular response to DNA double-strand breaks. Exp. Cell Res. 300, 320-334.

Bekker-Jensen, S., Lukas, C., Kitagawa, R., Melander, F., Kastan, M.B., Bartek, J., and Lukas, J. (2006). Spatial organization of the mammalian genome surveillance machinery in response to DNA strand breaks. J. Cell Biol. 173, 195-206.

Boulton, S.J., Martin, J.S., Polanowska, J., Hill, D.E., Gartner, A., and Vidal, M. (2004). BRCA1/BARD1 orthologs required for DNA repair in Caenorhabditis elegans. Curr. Biol. 14, 33-39.

Broude, N.E., and Budowsky, E.I. (1973). The reaction of glyoxal with nucleic acid components. V. Denaturation of DNA under the action of glyoxal. Biochim. Biophys. Acta 294, 378-384.

Chen, B.Y., Huang, C.H., Lin, Y.H., Huang, C.C., Deng, C.X., and Hsu, L.C. (2014). The K898E germline variant in the PP1-binding motif of BRCA1 causes defects in DNA Repair. Sci. Rep. 4, 5812.

Chin, G.M., and Villeneuve, A.M. (2001). C. elegans mre-11 is required for meiotic recombination and DNA repair but is dispensable for the meiotic G(2) DNA damage checkpoint. Genes Dev. 15, 522-534.

Craig, A.L., Moser, S.C., Bailly, A.P., and Gartner, A. (2012). Methods for studying the DNA damage response in the Caenorhabditis elegans germ line. Methods Cell Biol. 107, 321352.

Essers, J., Houtsmuller, A.B., van, V.L., Paulusma, C., Nigg, A.L., Pastink, A., Vermeulen, W., Hoeijmakers, J.H., and Kanaar, R. (2002). Nuclear dynamics of RAD52 group homologous recombination proteins in response to DNA damage. EMBO J. 21,
2030-2037.

Garcia-Muse, T., and Boulton, S.J. (2005). Distinct modes of ATR activation after replication stress and DNA double-strand breaks in Caenorhabditis elegans. EMBO J. 24, 4345-4355.

Godard, T., Deslandes, E., Sichel, F., Poul, J.M., and Gauduchon, P. (2002). Detection of topoisomerase inhibitor-induced DNA strand breaks and apoptosis by the alkaline comet assay. Mutat. Res. $520,47-56$.

Hofmann, E.R., Milstein, S., Boulton, S.J., Ye, M., Hofmann, J.J., Stergiou, L., Gartner, A., Vidal, M., and Hengartner, M.O. (2002) Caenorhabditis elegans HUS-1 is a DNA damage checkpoint protein required for genome stability and EGL-1-mediated apoptosis. Curr. Biol. 12, 1908-1918.

Hyun, M., Lee, J., Lee, K., May, A., Bohr, V.A., and Ahn, B. (2008). Longevity and resistance to stress correlate with DNA repair capacity in Caenorhabditis elegans. Nucleic Acids Res. 36, 13801389.

Hyun, M., Park, S., Kim, E., Kim, D.H., Lee, S.J., Koo, H.S., Seo, Y.S., and Ahn, B. (2012). Physical and functional interactions of Caenorhabditis elegans WRN-1 helicase with RPA-1. Biochemistry 51, 1336-1345.

Kalogeropoulos, N., Christoforou, C., Green, A.J., Gill, S., and Ashcroft, N.R. (2004). chk-1 is an essential gene and is required for an S-M checkpoint during early embryogenesis. Cell Cycle 3 , 1196-1200.

Langerak, P., and Russell, P. (2011). Regulatory networks integrating cell cycle control with DNA damage checkpoints and double-strand break repair. Philos. Trans. R. Soc. Lond B Biol. Sci. 366, 3562-3571.

Lankinen, M.H., Vilpo, L.M., and Vilpo, J.A. (1996). UV- and gamma-irradiation-induced DNA single-strand breaks and their repair in human blood granulocytes and lymphocytes. Mutat. Res. 352, 31-38.

Lee, S.J., Gartner, A., Hyun, M., Ahn, B., and Koo, H.S. (2010). The Caenorhabditis elegans Werner syndrome protein functions upstream of ATR and ATM in response to DNA replication inhibition and double-strand DNA breaks. PLoS Genet. 6, e1000801.

Lemmens, B.B., and Tijsterman, M. (2011). DNA double-strand break repair in Caenorhabditis elegans. Chromosoma 120, 1-21.

MacQueen, A.J., and Villeneuve, A.M. (2001). Nuclear reorganization and homologous chromosome pairing during meiotic prophase require C. elegans chk-2. Genes Dev. 15, 1674-1687.

Meza, J.E., Brzovic, P.S., King, M.C., and Klevit, R.E. (1999). Mapping the functional domains of BRCA1. Interaction of the ring finger domains of BRCA1 and BARD1. J. Biol. Chem. 274, 56595665.

Miyazaki, T., Bressan, D.A., Shinohara, M., Haber, J.E., and Shinohara, A. (2004). In vivo assembly and disassembly of Rad51 and Rad52 complexes during double-strand break repair. EMBO J. 23, 939-949.

Moynahan, M.E., Chiu, J.W., Koller, B.H., and Jasin, M. (1999). Brca1 controls homology-directed DNA repair. Mol. Cell 4, 511518.

Nakada, S., Yonamine, R.M., and Matsuo, K. (2012). RNF8 regulates assembly of RAD51 at DNA double-strand breaks in the absence of BRCA1 and 53BP1. Cancer Res. 72, 4974-4983.

Olive, P.L. (2011). Retention of gammaH2AX foci as an indication of lethal DNA damage. Radiother. Oncol. 101, 18-23.

Olive, P.L., and Banath, J.P. (2006). The comet assay: a method to measure DNA damage in individual cells. Nat. Protoc. 1, 23-29.

Olive, P.L., Banath, J.P., and Durand, R.E. (1990). Heterogeneity in radiation-induced DNA damage and repair in tumor and normal cells measured using the "comet" assay. Radiat. Res. 122, 86-94

Pachkowski, B., and Nakamura, J. (2013). A neutral glyoxal gel electrophoresis method for the detection and semi-quantitation of DNA single-strand breaks. Methods Mol. Biol. 1054, 133-143.

Paull, T.T., Rogakou, E.P., Yamazaki, V., Kirchgessner, C.U., Gellert, M., and Bonner, W.M. (2000). A critical role for histone H2AX in recruitment of repair factors to nuclear foci after DNA damage. Curr. Biol. 10, 886-895

Petalcorin, M.I., Galkin, V.E., Yu, X., Egelman, E.H., and Boulton, S.J. (2007). Stabilization of RAD-51-DNA filaments via an interaction domain in Caenorhabditis elegans BRCA2. Proc. Natl. Acad. Sci. USA 104, 8299-8304.

Polanowska, J., Martin, J.S., Garcia-Muse, T., Petalcorin, M.I., and 
Boulton, S.J. (2006). A conserved pathway to activate BRCA1dependent ubiquitylation at DNA damage sites. EMBO J. 25, 2178-2188.

Pommier, Y. (2006). Topoisomerase I inhibitors: camptothecins and beyond. Nat. Rev. Cancer 6, 789-802.

Powell, S.N., and Kachnic, L.A. (2003). Roles of BRCA1 and BRCA2 in homologous recombination, DNA replication fidelity and the cellular response to ionizing radiation. Oncogene 22 , 5784-5791.

Ryan, A.J., Squires, S., Strutt, H.L., and Johnson, R.T. (1991). Camptothecin cytotoxicity in mammalian cells is associated with the induction of persistent double strand breaks in replicating DNA. Nucleic Acids Res. 19, 3295-3300.

Scully, R., Ganesan, S., Vlasakova, K., Chen, J., Socolovsky, M., and Livingston, D.M. (1999). Genetic analysis of BRCA1 function in a defined tumor cell line. Mol. Cell 4, 1093-1099.
Singh, N.P. and Khan, A. (1995). Acetaldehyde: genotoxicity and cytotoxicity in human lymphocytes. Mutat. Res. 337, 9-17.

Sobkowiak, R., and Lesicki, A. (2009). Genotoxicity of nicotine in cell culture of Caenorhabditis elegans evaluated by the comet assay. Drug Chem. Toxicol. 32, 252-257.

Tarsounas, M., Davies, A.A., and West, S.C. (2004). RAD51 localization and activation following DNA damage. Philos. Trans. R. Soc. Lond B Biol. Sci. 359, 87-93.

van Veelen, L.R., Cervelli, T., van de Rakt, M.W., Theil, A.F., Essers, J., and Kanaar, R. (2005). Analysis of ionizing radiation-induced foci of DNA damage repair proteins. Mutat. Res. 574, 22-33.

Wicky, C., Alpi, A., Passannante, M., Rose, A., Gartner, A., and Muller, F. (2004). Multiple genetic pathways involving the Caenorhabditis elegans Bloom's syndrome genes him-6, rad-51, and top-3 are needed to maintain genome stability in the germ line. Mol. Cell Biol. 24, 5016-5027. 